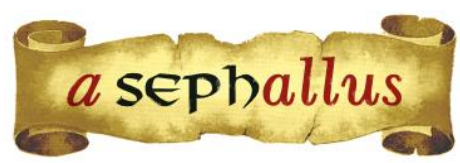

Revista aSEPHallus de Orientação Lacaniana

Núcleo Sephora de Pesquisa sobre o Moderno e o Contemporâneo

ISSN $1809-709 \mathrm{X}$

\title{
Les spécificités lacaniennes dans l'opposition psychose/autisme
}

\author{
Michel Grollier \\ Orcid: https://orcid.org/0000-0001-6844-8897 \\ Psychanalyste \\ Membre de l'École de la cause freudienne et de l'Association Mondiale de Psychanalyse (Paris, France) \\ Professeur à l'Université de Rennes 2 (Paris, France) \\ E-mail: michel.grollier@univ-rennes2.fr
}

\begin{abstract}
Resumé : Nous avons une approche de la clinique des enfants autistes autour d'un rapport singulier à la voix de l'Autre, c'est-à-dire le lieu d'origine des signifiants, qui s'incarne habituellement dans la mère et les quelques partenaires de l'enfant. La question étant ici, comme le dit Lacan, de voir d'où ils ont entendu ce qu'ils nous produisent. Ceci se lie à la clinique même de Kanner qui cherche d'où viennent les énoncés étonnants des autistes. Avec ce point de départ, nous présenterons les spécificités lacaniennes dans l'opposition psychose / autisme à partir des travaux de plusieurs psychanalystes d'importance dans le domaine.
\end{abstract}

Mots-Clés : Autisme; psychose; corps; voix; pulsion.

As especificidades lacanianas na oposição psicose/autismo: Temos uma abordagem da clínica das crianças autistas em torno de uma relação singular à voz do Outro, o que quer dizer o lugar de origem dos significantes, que se encarna habitualmente na mãe e em alguns parceiros da criança. A questão sendo aqui, como diz Lacan, a de ver de onde eles ouviram o que eles produzem para nós. Isto está em ligação com a própria clínica de Kanner, que procura de onde vêm os enunciados surpreendentes dos autistas. Tendo esse ponto de partida, apresentaremos as especificidades lacanianas na oposição psicose/autismo a partir dos trabalhos de diversos psicanalistas que se destacam na área.

Palavras-Chave: Autismo; psicose; corpo; voz; pulsão.

Lacanian specificities in the psychosis/autism opposition: We have an approach to the autistic children's clinic around a singular relationship to the voice of the Other, which means the place of origin of the signifiers, which is usually embodied in the mother and in some of the child's partners. The question being here, as Lacan says, is to see where they heard what they produce for us. This is in connection with Kanner's own clinic, which looks for where the surprising statements of autists come from. With this starting point, we will present the Lacanian specificities in the opposition psychosis / autism from the work of several important psychoanalysts in the area.

Keywords : Autism; psychosis; body; voice; drive. 
Revista aSEPHallus de Orientação Lacaniana

Núcleo Sephora de Pesquisa sobre o Moderno e o Contemporâneo

ISSN $1809-709$ X

\section{Les spécificités lacaniennes dans l'opposition psychose/autisme}

\section{Michel Grollier}

Avec Lacan il y a d'abord deux phrases, reprises plusieurs fois et dont la forme aboutie se trouve dans le texte L'Étourdit : «Qu'on dise reste oublié derrière ce qui se dit dans ce qui s'entend. Cet énoncé, qui paraît d'assertion pour se produire dans une forme universelle, est de fait modal, existentiel comme tel : le subjonctif dont se module son sujet, en témoignant » (Lacan, 1972/2001, p. 449). Le dire comme trace du sujet se trouve subverti par ce qui est dit en tant que c'est entendu. Comment ne pas penser là à la conversation de Lacan avec le Dr. Cramer durant la conférence sur le symptôme à Genève en 1975, Cramer prenant l'exemple des enfants autistes auprès desquels il intervient. Cramer reprend la question du côté de l'enfant, le « encore faut-il que l'enfant l'entende » à propos de la mère. Pour Lacan, entendre fait partie de la parole, il précise «Que vous souleviez la question qu'il y ait des êtres qui n'entendent rien est suggestif certes, mais difficile à imaginer » (Lacan, 1975/1985, p. 5). Cramer avance alors la question des enfants autistes et Lacan dit :

Comme le nom l'indique, les autistes s'entendent eux-mêmes. Ils entendent beaucoup de choses. Cela débouche même normalement sur I'hallucination, et I'hallucination a toujours un caractère plus ou moins vocal. Tous les autistes n'entendent pas des voix, mais ils articulent beaucoup de choses, et ce qu'ils articulent, il s'agit justement de voir d'où ils I'ont entendu. (Lacan, 1975/1985, p. 5).

Donc nous avons une approche de la clinique des enfants autistes autour d'un rapport singulier à la voix de l'Autre, c'est-à-dire le lieu d'origine des signifiants, qui s'incarne habituellement dans la mère et les quelques partenaires de l'enfant. La question étant ici, comme le dit Lacan, de voir d'où ils ont entendu ce qu'ils nous produisent. C'est là en lien avec la clinique même de Kanner qui cherche d'où viennent les énoncés étonnants des autistes.

Quand Cramer dit qu'ils n'arrivent pas à nous entendre, Lacan corrige: « Mais c'est tout à fait autre chose. Ils n'arrivent pas à entendre ce que vous avez à leur dire en tant que vous vous en occupez» (Lacan, 1975/1985, p. 5). C'est donc directement la place de partenaire, que suppose une énonciation de celui qui veut s'occuper de l'enfant, qui ne peut trouver d'adresse. Et quand Cramer explique que pour lui leur langage est fermé, Lacan (1975/1985, p. 18) reprend : « C'est bien justement ce qui fait que nous ne les entendons pas. C'est qu'ils ne vous entendent pas. Mais enfin, il y a sûrement quelque chose à leur dire ». Lacan (1975/1985) nous oriente donc vers les conditions nécessaires pour un dire qui emporte la place du sujet autiste sans le viser dans une interlocution qui supporterait notre demande. Et Lacan, qui soutient sa position sur I'humain 
comme être de langage, fait pour le langage dans son humanité même, va insister sur la question des autistes:

Il s'agit de savoir pourquoi il y a quelque chose chez l'autiste, ou chez celui qu'on appelle schizophrène, qui se gèle, si on peut dire. Mais, vous ne pouvez dire qu'il ne parle pas. Que vous ayez de la peine à entendre, à donner sa portée à ce qu'ils disent, n'empêche pas que ce sont des personnages finalement plutôt verbeux (Lacan, 1975/1985, p. 18)

Avec Lacan, nous avons donc un point qui fait obstacle à la parole, quelque chose qui se gèle, et ce qu'ils produisent dans la langue est difficilement recevable car non articulé comme énonciation qui nous soit adressée, dont nous soyons partenaires. Que ce soit le S1 qui fait accroche du sujet dans l'appareillage du langage (comme nous l'avons vu) est une des hypothèses privilégiées dans le champ freudien. Ou plutôt même une pétrification dans le S1 qui reste alors sans possible liaison dans un S2. C'est une des propositions de Rosine et Robert Lefort, qui ont représenté depuis leur participation au séminaire de Lacan un courant important de l'accueil des enfants autistes et psychotiques dans la suite de Lacan. Cette conception s'appuie sur la construction du cri chez l'enfant, première élaboration sonore de l'enfant, qui doit rencontrer dans la réponse de l'Autre, I'Autre maternel, sa transformation en appel. Cette transformation fait du cri dans l'après-coup, un S1 articulé au S2 de sa réponse. Lacan, déjà interpellé du côté du cri lors de la conférence sur le symptôme avait dit « Freud parle du cri à un moment. Il faudrait que je vous le retrouve. Il parle du cri, mais cela tombe à plat. » (Lacan, 1975/1985, p. 20). Il fait là référence à un passage de l'Esquisse de Freud.

Dans sa réflexion, Lacan a poursuivi son apport à la question des discours comme modalité du lien social, mais bien évidemment, dans la schizophrénie et l'autisme, nous sommes hors discours, même si le sujet qui vire à la paranoïa, à l'aide notamment du délire, tente de forger un semblant de discours qui fasse un lien possible. J.-A. Miller, dans un article sur ce qu'il nomme Clinique Ironique, spécifie avec Lacan cette singularité du schizophrène : il ne se défend pas du réel au moyen du symbolique, plus précisément : «il ne se défend pas du réel par le langage, parce que pour lui le symbolique est réel »(Miller, 1993, p. 7). Ainsi, Miller montre la pente ironique du schizophrène. Pour lui, elle est en somme une tentative de dire quelque chose du monde, dire que l'Autre n'existe pas, que le lien social est en son fond une escroquerie. Miller poursuit : «Dans la perspective schizophrénique, le mot n'est pas le meurtre de la chose, il est la chose » (Miller, 1993, p. 9). Ce qui fait néanmoins l'ancrage de la psychose dans le monde, c'est d'être sûr de «la chose », au sens freudien. Et nous pouvons en voir les déclinaisons entre mélancolie, paranoïa et schizophrénie. Nous comprenons bien avec ces éléments pourquoi dès ses 
premiers travaux, Kanner a eu tendance à rapprocher l'autisme infantile de la schizophrénie, et le choix même de la dénomination.

Pour aborder ce monde et faire consister une présence qui puisse s'adresser à un autiste, R. et R. Lefort propose d'inverser la proposition de Lacan : «il y a quelque chose qu'on ne peut pas leur dire» (Lefort \& Lefort, 1997). Estéban Morilla (2001), dans son article de Mental, relève quelques difficultés dans les positions proposées par les Lefort. Une structure sans sujet et dont le débouché est le passage du côté d'une «psychotisation ». Une possibilité pour contourner ces difficultés est la reprise d'une formule, déjà abordée par E. Laurent en 1992, et relancée par P. Naveau lors des XXII ${ }^{\text {ìme }}$ journées du CEREDA consacrée à «L'enfant et ses joies » (comme relevé par E. Morilla), la forclusion du signifiant du désir de la mère (DM). Absence donc de la possibilité même de symboliser un désir de la mère pour l'enfant. Forclusion anticipatrice qui laisse l'enfant face à une omnipotence réelle. Morilla relève que pour $\mathrm{R}$. et $\mathrm{R}$. Lefort l'enfant autiste est hors langage plus que hors discours. En 2003, Robert et Rosine Lefort ponctueront leur parcours en prenant l'option de proposer une structure autistique, structure où l'Autre n'existe pas. Ouverture qui, dans une lecture du séminaire de J. A. Miller et E. Laurent sur L'Autre qui n'existe pas et ses comités d'éthique, les conduit à un questionnement élargi sur la civilisation. Leur thèse ainsi se singularise dans cette position qui ne propose pas d'Autre au sujet, mais un double réel. Ce travail a su susciter dans le champ freudien une conversation nécessaire pour constituer une dynamique de recherche et d'élaboration, qu'il ne faudrait pas laisser s'éteindre.

A la suite des Lefort ${ }^{1}$, dans le champ lacanien, Maleval s'est attaché à l'étude de ces solutions autistes et à la façon dont ces petits sujets traitent leur rapport à celui-ci. Déjà dans les années quatre-vingt-dix, Eric Laurent (1992), dans une rencontre du CEREDA 2 à Toulouse, avait construit une discrimination du traitement de la jouissance (c'est-à-dire des manifestations de la vie dans le corps, de toute ces tensions et pulsions qui animent la vie) non insérée dans un ordre symbolique (donc non réglé par l'ordre symbolique courant fondateur de la civilisation). Il proposait que la jouissance fasse alors retour soit dans l'Autre (délire), soit dans le corps (dissociation) soit sur un bord (objet ou assimilé). Cette différence de traitement de la jouissance n'est pas en elle-même discriminatoire entre psychose et autisme, mais Maleval va reprendre la proposition des Lefort pour justifier un écart, dans le traitement de l'objet et du langage, dans les constructions imaginaires.

D'abord dans l'opération même qui institue depuis Freud la psychose, opération que Lacan avait écrite comme forclusion du signifiant du Nom du père. Pour que cette opération s'applique, il y faut déjà une articulation signifiante. L'autiste se tenant à I'orée de l'Autre comme lieu des signifiants, manifeste là une résistance aux couleurs de refus, refus référé à ce support sonore qui s'objective comme voix dans le monde humain (ce que les études les plus scientifiques confirment ${ }^{3}$ ). Réduire la voix au rang de bruit ne leur épargne pas vraiment le choc de ses effets, 
car c'est dans ce lieu que se profile une réponse possible aux mouvements de la vie qui les habite. A ne pas céder à cette offre, les humains qui l'entoure pousse l'autiste à trouver une voie pour s'en saisir. Mais il s'agira toujours dans ce cas d'un nouvel après coup, sans cette morsure dans le corps qui en tant que marque réelle, inscrite hors de portée du sujet, le lie à ce corps qui l'incarne. D'où cette pente, reprise par quelques auteurs, à envisager une forclusion de l'Autre en tant que lieu des signifiant, y compris incarné dans les partenaires parentaux. Une opération du même type mais sur un autre objet. Sauf que, se faisant sujet, l'autiste se plonge d'une façon ou d'une autre dans le signifiant.

C'est une question clinique centrale. Nous retrouvons là un certain nombre d'enfant, de présentation «pré-kannerienne » comme dirait Maleval, et qui pourtant ont par phase utilisé le langage de manière très performante, dans un certain rapport à un partenaire, partenaire au départ imaginaire, qui ont reflué sur des positions de nouveau très pauvres. J'avais observé des phénomènes du même type dans la schizophrénie, certains sujets stabilisés pouvant, devant une rencontre aléatoire impossible chuter dans une catatonie profonde, corps déshabité. Des sujets, que les infirmiers m'avaient appris à accompagner, à défaut de traitements efficaces, par le partage de conversation concernant le sujet au pied de son lit. Surprise alors du retour brutal du sujet dans la conversation (dans le meilleur des cas).

Là, I'accrochage signifiant avait fait son effet. Mais avec les autistes l'appel du signifiant ne fonctionne quasiment jamais. À la place, c'est à l'imaginaire qu'il faut faire appel, un imaginaire peu à peu scénarisé afin de lui donner une pente symbolique. Prendre appui sur l'objet de l'enfant, le prendre en compte et s'en faire partenaire, pour, par déplacement, être ce qui fait office de partenaire pour ces sujets perdus qui peuvent alors s'accrocher à ce que nous mettons à disposition. Et par la suite évidemment se montrer un peu réticent, difficile, l'art étant de le faire avec le tact nécessaire pour ne pas rompre le lien et introduire la rencontre à un effet symbolisant, qui permettra au sujet de traiter le monde à partir de là. Ou encore de suivre le fil des constructions qu'a déjà su élaborer l'enfant, jeux imaginaires avec des personnages, personnages qui prêtent leurs voix à celui qui ne la supporte pas.

Il y a donc bien un écart là avec la psychose, car même si le support signifiant est primordial aux effets de lien social, même si nous les tirons de ce côté, les autistes n'y consentent qu'au prix de le traiter à leurs façons, sans toujours en accepter les contraintes, et surtout l'équivoque. Nulle ironie chez l'autiste, contrairement au schizophrène (Miller, 1993), nul jeu de signification! C'est le paradoxe pour ces sujets réputés en rupture de lien, ils sont trop liés à une signification, à la dimension du signe. C'est aussi pour ça que les accompagnements spécifiques basé sur pictogramme et de signes, ont une certaine efficacité pour eux. Pas besoin de demande et d'équivoque ! Mais c'est aussi un problème, car, à suivre les signes, ils ne peuvent rien savoir de l'incertitude du monde, de ses ratages. Et d'où aussi l'insupportable du ratage pour ces sujets si 
minutieux... Pour l'autiste une demande doit correspondre à une réponse corrélée, ils sont d'ailleurs très doués en encodage. Le risque alors est celle du trop, le symptôme propre à l'autiste c'est le trop, trop de vie, trop de demandes, trop de bruit... Tout l'inverse du psychotique pour lequel il y a toujours l'inquiétude de la question qui ne pourrait viser rien d'autre que leur être, et qui sont susceptible de noyer l'autre sous des flots de revendications.

\section{L'originalité de Maleval et la thérapie par affinité.}

Cela renvoie aussi à la conception de la psychose. Maleval nous parait être celui qui, dans la suite des intuitions des Lefort, s'est cliniquement intéressé assez vite à la distinction entre schizophrénie et autisme. Il s'est penché sur la question très tôt et, en 1998, proposait que I'autisme n'évoluait pas vers la psychose, mais vers l'autisme (Maleval, 1998). Il propose dans ses travaux de différencier autisme et psychose par la clinique :

Les caractéristiques les plus évidentes de la psychose ne se discernent guère dans l'autisme ; pourtant ils partagent en commun des troubles de l'identité, du cours de la pensée et des phénomènes de délocalisation de la jouissance qui conduisirent longtemps à souligner le recoupement partiel de leurs deux cliniques (Maleval, 2014, p. 170).

Mais aussi une différence par l'histoire: «C'est la schizophrénie de Bleuler qui sert de référence à Kanner et à Asperger quand ils dégagent le syndrome autistique ; c'est pourquoi l'un et l'autre vont chercher dans le vocabulaire de Bleuler le terme par lequel tous deux nomment leur découverte sans s'être concertés et sans connaître leurs travaux » (Maleval, 2014, p. 170). Enfin dans le fil de la remarque faites plus haut, il repère combien l'ironie schizophrénique est antagoniste de l'immuabilité autistique (Maleval, 2014).

Il énumère ainsi dans un travail en trois parties, publié dans les années 2014-2015, son hypothèse de structure autistique [4] par toute une série de faits contradictoire entre les positions psychotique et autistique qui marque des accompagnements à nécessairement différencier. Traitement de l'objet, du partenaire, du signe, de la règle et du signifiant. Enfin il reprend l'un des éléments mis en avant par tous les cliniciens et chercheurs, l'âge du démarrage des troubles. C'était d'ailleurs déjà un marqueur pour Kanner, et ça le reste, l'enfant autiste manifeste d'emblée son décalage au partenaire et au monde en général, c'est notamment ce qui entretien l'espoir de réduire cette différence à une atteinte organique. Nul déclenchement donc dans l'autisme, autre grande différence qui signe que nous n'avons pas affaire à un échec après une inscription dans un parcours, mais à un départ empêché dirais-je. Enfin un large consensus des auteurs modernes nous rappelle la quasi absence de passage de l'autisme à la schizophrénie (Maleval, 2014). 
Tous ces travaux l'on conduit à nous proposer, comme l'avaient envisagé en leur temps Rimland et Kanner, mais pas pour les mêmes raisons, que l'autisme soit une structure clinique à part entière. Pour théoriser cela Maleval prend appui sur quelques points. L'objet bien sûr, dont il fait le partenaire de l'autiste et support d'un imaginaire qui peut s'étendre à son monde. Et dans cet ensemble l'objet voix dont le traitement parait être l'un des points de départ du positionnement autistique. Son travail sur la langue dans l'autisme (Maleval, 2011) lui permet de spécifier l'usage si particulier qu'en fait l'autiste, et situe ce dernier dans une perspective bien différente du psychotique qui dans la voix trouve l'objet de sa persécution. Ainsi avec la survenue d'énonciation surprenante chez des sujets autistes autrement mutique, il trouve la démonstration que l'autiste a bien un certain rapport à l'Autre, mais un rapport des plus douloureux qu'il ne peut que rejeter à défaut de le contenir. La thèse de Maleval est ainsi que :

Il [l'autiste] n'est pas resté au bord de l'aliénation, il est bien dans l'aliénation, mais il la refuse. L'aliénation signifiante n'est pas assumée par l'autiste. Il n'y a pas aphanisis du sujet, en revanche le langage fait écho dans le corps ; de sorte que Jacques-Alain Miller suggère d'utiliser le terme de parlêtre pour désigner l'autiste » (Maleval, 2011, p. 161.).

C'est donc dans ce rapport si étrange (pour nous) au signifiant que l'autiste se loge, où le mot n'est pas totalement le meurtre de la chose, et donc où le semblant est déficient. Nous saisissons là ces rencontres avec de jeunes autistes qui, éduqués, citent le mot devant l'image de la chose, mais sans jamais auparavant avoir trituré le signifiant pour s'en approcher. Telle petite fille, ayant bénéficié de l'accompagnement précoce et intensif d'une rééducation orthophonique, savait répondre chien devant l'image du chien, vache devant l'image de la vache, sans jamais avoir usé de «ouah » ou de «meuh » ou de tout autre manipulation du signifiant, et qui bien sûr n'en usa jamais. Nous y reviendrons.

D'autres auteurs lacaniens, sans construire un modèle d'accompagnement comme Maleval avec la thérapie par affinité, ont proposé des interprétations de la question autistique.

\section{Laznik et Preaut.}

M. C. Laznik et sa collègue G. Gabassu ont mis en place des séminaires qui visent à articuler les thèses sur le ratage de la structure (en s'articulant avec des équipes de PMI) et les difficultés des familles. La recherche PREAUT [5] qu'ils ont initiée donne lieu depuis des années à la publication d'une revue, «Les Cahiers de Preaut »[6] I'un des objectifs étant de tenter de valider des signes prédicteurs d'autisme chez l'enfant de moins de 2 ans. Ce courant s'écarte donc des deux précédents dans sa logique, mais s'oriente d'une même référence à une lecture de Lacan. Voyons maintenant quelques-unes de ces thèses. 
S'intéressant aux tout-petits et au risque autistique, Marie-Christine Laznik (1996/2003), reprenant des apports de Colette Soler et en se référant à Lacan, notamment à partir du Séminaire $\mathrm{XI}$, évoque que dans la constitution du sujet il y a deux temps, l'aliénation et la séparation (interview paru en novembre 2005 sur le site Edipe pour la parution des Cahiers de Préaut). C'est ce temps de l'aliénation qui manquerait dans la constitution du sujet autistique. Il y aurait ainsi un arrêt dans le circuit pulsionnel de la voix. Ce qui oriente le traitement, c'est qu'un psychanalyste puisse arriver à remettre en route ce circuit pulsionnel en jouant avec le bébé et sa mère. Dans ce lien transférentiel, la mère peut retrouver une voix porteuse de la «pulsion invoquante » que I'indifférence de son bébé avait éteint. Marie-Christine Laznik (1996/2003) essaie de démontrer dans ses recherches qu'il y a certaines dimensions prosodiques et rythmiques auxquelles nul ne peut se soustraire, pas même le bébé à risque autistique.

Elle pose qu'il y aurait ainsi un arrêt dans le circuit pulsionnel de la voix. Elle conjoint donc l'objet voix comme non institué de par l'impossible organisation pulsionnelle à l'absence d'aliénation ; articulant l'absence d'aliénation au signifiant avec la non-institution de la voix comme objet humain. Marie-Christine Laznik fait pour cela référence à des études récentes de neurosciences et de physiologie. Les chercheurs ayant repéré que le jeune autiste ne discriminait pas la voix humaine des autres sons, contrairement aux autres enfants (Zilbovicus, 2005).

Elle raisonne ainsi en écho avec les travaux de neurobiologie. Si le bébé répond à la voix humaine, cela active des zones cérébrales qui, dans le développement de l'autiste, ne sont pas normalement activées et périclitent. Un possible abord thérapeutique, consisterait à chercher à entrer en relation avec l'enfant susceptible de devenir un enfant autiste, en jouant sur cette dimension de la voix dans le lien à la mère._A partir de l'expérience du séminaire du centre $A$. Binet animé par $B$. Touati, un ouvrage récent propose un certain nombre d'exemple thérapeutique de ce travail sur la voix et le langage (Touati, 2007).

Mais Laznik, influencé par la thèse d'une collègue brésilienne (Vocaro, 2009), en viendra aussi à tenter de différencier autisme et psychose à partir de ce qui s'élabore entre réel et réalité, dans le nouage des registres réel, imaginaire et symbolique. Elle a ainsi produit un intéressant chapitre en 2016, ou elle tente de distinguer psychose et autisme chez le tout petit. Sa démonstration suit le tressage des trois registres RSI et les ratages qui peuvent venir empêcher le nouage borroméen mis en évidence par Lacan. De fait son hypothèse est en deçà, puisqu'elle part du postulat que c'est l'imaginaire qui est touché chez le petit sujet autiste et que c'est ce « brin » qui va très rapidement rater son accroche sur les deux autres registres. De là l'écart qu'elle démontre, tant avec Joyce (dont elle s'est inspirée) que de la psychose dont elle propose plusieurs types de ratages. Elle écrit ainsi : 
Il est courant de convenir que les enfants autistes ont un défaut du champ de l'Imaginaire. Non seulement le corps ne tient pas avec les autres instances mais il leur est généralement difficile d'imaginer des histoires. Même les autistes de haut niveau pâtissent de cette difficulté. Cela leur offre parfois la possibilité de traiter le Symbolique avec le Réel sans s'encombrer des dimensions Imaginaires. Cela peut donner d'excellents ingénieurs. Mais dans la vie au jour le jour, cela ne facilite pas le contact avec les autres (Laznik, 2016, p. 428).

Sur le fait qu'il leur est difficile d'imaginer des histoires, la question se pose, comme pour Dona Williams par exemple et d'autres. Mais la piste est intéressante puisqu'elle rappelle qu'elle prend le réel du côté de l'organique (la vie que pointe Freud (1950[1895]/2011) dès l'Esquisse, comme dit plus haut), le symbolique comme l'ordre du monde (et là avec Freud et Lacan nous positionnons le langage), et pour l'imaginaire, elle dit «L'Imaginaire sera la possibilité de voir ce qui n'est pas encore advenu, His Majesty the Baby, là où il n'y a pour l'instant qu'un petit organisme bien fragile » (Laznik, 2016, p. 428). Cet imaginaire primitif pose question dans la logique lacanienne. En effet Lacan se saisit tant de l'étude de Freud sur le narcissisme que de ce qu'il a élaboré comme stade du miroir. Le registre de l'imaginaire chez Lacan renvoie à l'image du corps, registre du leurre et de l'identification, le registre qui vient perturber et subvertir la rencontre des autres. Le stade du miroir est pour Lacan moment fondateur d'une unité imaginaire qui rester aliénante tout en permettant au petit être de se positionner vis-à-vis de l'Autre. C'est I'approche lacanien du narcissisme. Cet imaginaire viendra constamment parasiter le sujet dans son rapport au désir ou plus radicalement a son corps. C'est aussi pourquoi dans la schizophrénie nous aurons cette difficulté avec le corps et son unité. Dans l'imaginaire nous trouvons une certaine continuité alors que le symbolique introduit la rupture, l'opposition qui fonde le code. Il est ainsi difficile d'accéder à l'imaginaire en l'absence d'un nouage au symbolique. Et les deux registres du symbolique et de l'imaginaire s'efforce, en s'y nouant, de recouvrir de sens le réel.

Sans vouloir rajouter de la difficulté, et même en y trouvant des écarts, il est clair que I'effort de Laznik rejoint celui de Maleval et des Lefort avant dans la distinction de l'autisme (Lefort \& Lefort, 2003). Si chez les Lefort persistait une ambiguïté sur cette différence avec la schizophrénie, à les prendre au sérieux ces auteurs ont séparé l'autisme de la psychose en général.

Rajoutons qu'avec I'association PREAUT les psychanalystes et les chercheurs travaillant avec Laznik ont développés certains outils. Dans une étude multicentrique coordonnée par le $\operatorname{Pr} C$. Bursztejn du CHRU de Strasbourg, 27 items signalés dans la littérature comme susceptible d'annoncer l'apparition d'un trouble autistique ont été testés chez 2350 enfants au cours d'examens de santé systématiques entre 8 et 13 mois. Au terme de cette étude, 8 de ces items, 
dont la présence a pu être attesté chez au moins $95 \%$ des bébés sains examinés, ont été jugés suffisamment fiables pour être utilisé à cet âge : ils constituent le « questionnaire sur le développement de la communication » (QDC). L'équipe Préaut, en prenant en compte les études sur les interactions précoces, et notamment ceux de C. Trevarthen et de son équipe (Aitken \& Trevarthen, 2003), a donc décidé de coupler ses recherches avec celle du Pr Bursztejn. L'idée présentée par G. C. Crespin et J. L. Sarradet a été de regrouper et valider l'ensemble de ces indices pour le dépistage des troubles du développement. Partant d'un premier outil international, le CHAT [7] , le même protocole global a ainsi été testé et adapté dans le cadre de la recherche Préaut et est désormais répendu dans les centres de dépistage.

\section{Autres repères lacaniens}

En s'interrogeant sur cet univers spécifique dans lequel se positionne le sujet autiste, espace qui a induit les réflexions sur ses dimensions (2 seulement ?), sur sa temporalité, sur sa dimension trouée ou centrée sur une enveloppe ou une carapace, se sont développé des réflexions topologiques. C'est ainsi qu'un autre chercheur, Gilles Mouillac (2014) a aussi réalisé une thèse sur la topologie de l'autisme. Cela entraine des questions sur l'insertion du discours de la psychanalyse dans le champ social, la question de sa transmission, qui doit se jouer plus sur des propositions d'accompagnement que sur ses élaborations. Et pas uniquement avec ces textes très technique, parfois aussi avec des travaux faisant appel à l'imaginaire. Ainsi Sauret, qui a beaucoup travaillé sur la question de l'autisme peut écrire en 2019 :

Pour certains milieux universitaires et intellectuels, mais également pour une partie de nos contemporains, la psychanalyse est devenue incompréhensible ; j'avoue que certains développements de Meltzer, par exemple, me paraissent tout simplement délirants : les psychanalystes seraient plus menacés par l'autisme à plusieurs que par l'autisme à deux, dont Lacan suspectait la cure. (Sauret, 2019, p. 113)

Sauret fait une analyse très ancrée dans le symbolique avec un axe sur le langage et les énoncés étranges des autistes, reprenant comme Grollier les travaux de Kanner.

S'inspirant de Pierre Bruno il s'interroge, comme Maleval sur la constitution chez le sujet autiste d'un Autre de synthèse, ce qui viendrait tenter d'inclure le sujet dans un certain ordre du langage. C'est la question du refus ou du manque d'un signifiant ayant mordu sur la vie du sujet, ce qui fait jouissance en son corps, et le propulsant dans la logique signifiante. En place de ce S1, il y aurait usage d'objets ou de tout autre instrument qui articule le sujet aux autres, qui autorise une certaine circulation de cette, de ces jouissances qui ne cessent pas avec la vie. Sur le rôle de I'objet parfois ici aussi les positions diffèrent dans leurs traitements. Ainsi Sauret (2019): «Il est 
une position que je veux stigmatiser : celle qui consiste, sous prétexte de « traitement de l'Autre », à s'adresser aux objets que le sujet n'arrive pas à intégrer au monde qu'il construit et dont l'extériorité semble le persécuter » (p. 114). Alors que les premiers psychanalystes s'inquiétaient de ce qui semblait dans l'objet renforcer la coupure ou le retrait du monde, ce qui conduisit Tustin à considérer que l'enfant autiste a affaire à un monde dangereux incarné tant dans de mauvais objets que de dangereux partenaires. Mais l'expérience a montré leur utilité, à défaut de mieux, pour les sujets autistes. Reste que le débat persiste entre ceux qui pousse à déshumaniser l'objet pour accompagner l'enfant vers l'être parlant, et ceux qui en use comme l'autiste en pariant sur un déplacement à venir. Comme le rappelle Golse (2006, p. 457): « Mais cela rejoint au fond ce que disait F. Tustin de l'objectif de toute thérapeutique d'enfant autiste : faire sentir à l'enfant qu'un autre existe, et qu'il n'est pas dangereux...tout simplement ! ».

Il faut aussi citer Juan Pablo Lucchelli. Psychanalyste, psychiatre et père d'un enfant autiste, il travaille en institution hospitalière et a écrit notamment Autisme. Quelle place pour la psychanalyse ?(Lucchelli, 2018). Précisons qu'il est donc psychiatre, mais a un doctorat de psychanalyse, un doctorat de psychologie et un doctorat de philosophie, ce parcours montrant sa quête dans le savoir pour interroger la condition humaine. Luccheli prend une position claire, l'autisme est différent de la psychose (de la schizophrénie) et note combien, pour une fois, le DSM a entériné un point qui a fait difficulté pour la psychiatrie européenne. L'autisme n'est pour lui ni déficitaire, ni une psychose, et si l'autisme est bien un trouble neurobiologique déterminant un rapport différent à la réalité, il s'agit de les accompagner au mieux dans leurs inventions, et c'est là que pour lui, la psychanalyse peut trouver sa place.

\section{Notes:}

1. Notamment à partir de leur ouvrage : Lefort R.et R. La distinction de l'autisme. Paris: Seuil; 2003.

2. CERADA : Centre d'Etude et de Recherche sur l'Enfant dans le Discours Analytique.

3. Gervais H. et coll. Recherche avec IRM mené par l'équipe de l'université de Tours intitulé «La reconnaissance de la voix par les sujets autistes », qui converge avec les travaux de M. Zilbovicius dans la même équipe.

4. Travail publié dans les revues La Cause freudienne numéros 23, 24 et 25 en 2014 et 2015.

5. Association PREvention AUTisme, qui publie régulièrement des Cahiers et contribue à des recherches, et a des accueils d'enfants autistes - https://www.preaut.fr/lassociation-fr/.

6. Les cahiers de Preaut, Revue annuelle de l'association Preaut, Éres, Toulouse.

7. Le Checklist for Autism in Toddlers (le CHAT), test initialement mis au point et validé en 1992. Simon Baron-Cohen et ses collègues, qui ont élaboré le CHAT, ont compilé des 
données sur 16000 enfants du sud-est de l'Angleterre auxquels on avait fait faire le test. Cette épreuve de prédictivité a été plusieurs fois évalué et paraît donner des résultats interessants (S. Baron-Cohen, J. Allen, C.Gilliberg). Il s'agit d'un questionnaire généraliste passable par la famille auprès d'un médecin généraliste. Le C.H.A.T. est constitué de deux questionnaires : I'un réservé aux parents, l'autre au médecin ou travailleur social.

\section{Références Bibliographiques}

Aitken, K. \& Trevarthen, C. (2003). L'organisation soi/autrui dans le développement psychologique humain. La psychiatrie de l'enfant, 46(2), 471-520.

Ansermet F. (1997). Entretien avec R. et R. Lefort: Sur l'autisme, travaux et recherche en cours. Archives de psychanalyse, l'enfant prêt à porter. Paris : Éditions Agalma.

Crespin, G. C. \& Sarradet, J. L. Évaluation d'un ensemble cohérent d'outils de repérage des troubles précoces de la communication pouvant présager un trouble grave du développement de type autistique La recherche Préaut. Journal français de psychiatrie (25), 46-48.

Golse, B. (2006). À propos des stéréotypies chez les enfants autistes. Entre recherche et évitement de l'objet ou entre reprise développementale et dévitalisation. La psychiatrie de l'enfant, 49, 443-458.

Freud, S. (2011). Esquisse d'une psychologie scientifique. In: Nouvelle Traduction Bilíngue. Toulouse: Érès. (Travail Original de 1950[1895]).

Lacan, J. (2001). L'étourdit. In Autres écrits. Paris: Seuil. (Travail Original de 1973).

Lacan, J. (1985). Conférence de Genève sur Le symptôme. Le Bloc-notes de la psychanalyse, 5, 523. (Travail Original de 1975).

Laurent, E. (1992). L'autisme et la psychanalyse. Toulouse: Presses Universitaires du Mirail.

Laznik, M.-C. (2003). Vers la parole, trois enfants autistes en psychanalyse. Paris: Denoel, Espace Analytique. (Travail Original de 1996).

Laznik, M.-C. (2016). Peut-on penser une clinique du nœud borroméen qui distingue psychose et autisme chez le tout-petit ?. In Bergès-Bounes, M. et al (Org.). Les psychoses chez l'enfant et l'adolescent (pp. 415-437). Toulouse : ERES

Lefort, R. \& Lefort, R. (2003). La distinction de l'autisme. Paris: Seuil, Champ Freudien.

Lucchelli, J. P. (2018). Autisme. Quelle place pour la psychanalyse?: Édition Michele.

Maleval, J.-C . (1998). De l'autisme de Kanner au syndrome d'Asperger. L'Évolution psychiatrique, 3(63), 293-309.

Maleval, J.-C. (2011). Langue verbeuse, langue factuelle et phrases spontanées chez l'autiste. La Cause freudienne, 78, 77-92. 
Maleval, J-C. (2014). Pourquoi I'hypothèse d'une structure autistique ? (I). La Cause Du Désir, $87(2)$.

Maleval, J-C. (2014). Pourquoi I'hypothèse d'une structure autistique? (II). La Cause Du Désir, $87(3)$.

Miller, J.-A. (1993). Clinique Ironique. La Cause Freudienne, Revue de psychanalyse, 23, 5-13.

Morilla, E. (2001). L'autisme: des positions divergentes dans la psychanalyse. Mental, Revue internationale de psychanalyse appliquée, 11.

Mouillac, G. (2014). Topologie de l'autisme. (Thèse de Doctorat). Université Rennes 2.

Sauret, M.-J. (2019). In Bruno, P. et al. La différence freudienne. (pp. 113-120). Toulouse: ERES | Poche - Psychanalyse.

Touati, B., Joly, F. \& Laznik, M. C. (2007). Langage, voix et parole dans l'autisme. Paris : PUF le Fil Rouge.

Vorcaro, A. (2009). Topologia da formação do inconsciente: o efeito sujeito. Estudos Lacanianos, $3,45-62$.

Zilbovicus, M. (2005). Imagerie cérébrale et autisme infantile. In: Berthoz, A, Andres, C., Barthélemy, C, Massion, J. \& Rogé, B. (Org.). L'autisme, de la recherche à la pratique. Paris: Odile Jacob.

Citação/Citation: Grollier, M. (mai. 2019 a out. 2019). Les spécificités lacaniennes dans I'opposition psychose/ autisme. Revista aSEPHallus de Orientação Lacaniana, 14(28), 20-32. Disponível em www.isepol.com/asephallus. Doi: 10.17852/1809-709x.2019v14n28p20-32

Editor do artigo: Tania Coelho dos Santos.

Recebido/Received: 02/08/2019 / 08/02/2019.

Aceito/Accepted: 04/10/2019 / 10/04/2019.

Copyright: (c) 2019 Associação Núcleo Sephora de Pesquisa sobre o moderno e o contemporâneo. Este é um artigo de livre acesso, que permite uso irrestrito, distribuição e reprodução em qualquer meio, desde que o autor e a fonte sejam citados/This is an openaccess article, which permites unrestricted use, distribution, and reproduction in any medium, provided the author and source are credited. 